\title{
Esquemas Tradicionales de Roles Sexuales de Género, Poder en las Relaciones y Violencia en el Noviazgo
}

\section{Jorge Arturo Martínez-Gómez*1, Yolima Bolívar-Suárez, César Armando Rey- Anacona', Laura Camila Ramírez-Ortiz', Ana María Lizarazo-Ojeda' y Libia Yanelli Yanez-Peñúñuri' ${ }^{2}$}

'Universidad Pedagógica y Tecnológica de Colombia

2Universidad de Sonora

- Recibido: 07 - 10 - 2020 - Aceptado: 05 - 12 - 2020 . Avance online: 18 - 12 - 2020

RESUMEN. El presente estudio tuvo por objetivo evaluar la relación entre el doble estándar sexual, el poder en las relaciones sexuales, las actitudes hacia los roles de género y la violencia en el noviazgo, y realizar una comparación entre una muestra de adolescentes y jóvenes colombianos y mexicanos, mediante un estudio observacional-analítico transversal con un diseño correlacional-comparativo. Participaron 2,698 jóvenes y adultos con edades de 14 a 47 años $(M=20,15 ; D T=2,73)$ pertenecientes a los países de Colombia (59,6\%) y México $(40,4 \%)$. Los resultados mostraron que los participantes de Colombia y México utilizan violencia en el noviazgo de forma bidireccional. Asimismo, en ambos países las actitudes sexistas, el doble estándar sexual y el poder en las relaciones se correlacionaron de manera directa y significativa con varios tipos de violencia recibida y ejercida. Los resultados enfatizan la importancia del estudio de las relaciones de las actitudes y conductas tradicionalistas de género con la violencia en el noviazgo, para ser incluidas en futuras investigaciones para el diseño de programas de prevención de los malos tratos en el noviazgo.

PALABRAS CLAVE: Rol sexual de género, Poder en la relación, Violencia en el noviazgo, Adultos, Jóvenes.

\section{Traditional Schemes of Sexual Gender Roles, Power in Relationships and Dating Violence}

ABSTRACT. The present study aimed to evaluate the relationship between the double sexual standard, power in sexual relationships, attitudes towards gender roles and dating violence, and a comparison between a sample of Colombian adolescents and young people and Mexicans, through an observational-analytical cross-sectional study with a correlational-comparative design. 2,698 young people and adults aged 14 to $47(M=20.15, S D=2.73)$ from the countries of Colombia (59.6\%) and Mexico (40.4\%) participated. Participants from Colombia and Mexico were found to use dating violence in a bidirectional way. Likewise, in both countries, sexist attitudes, double sexual standards and, power in relationships were directly and significantly correlated with various types of violence received and practiced. The results emphasize the importance of studying the relationships of traditionalist gender attitudes and behaviors with dating violence to be included in future research to design programs for the prevention of mistreatment in dating.

KEYWORDS: Sexual gender role, Power in the relationship, Dating violence, Adults, Youth.

Los estereotipos sociales podrían incidir en que los adolescentes perciban que existen conductas en las relaciones de pareja mediadas por los roles de género, por lo tanto, se podría pensar que existen comportamientos que son

\footnotetext{
*Correspondencia: Jorge Arturo Martínez-Gómez.

Universidad Pedagógica y Tecnológica de Colombia

Dirección: Tunja, Colombia.

E-mail: jorgearturo.martinez@uptc.edu.co
}

(๑) 2021 Sociedad Universitaria de Investigación en Psicologíay Salud. Publicado porConsejo General de Colegios oficiciales de Psicólogos, España. Este es un articulo Open Access
bajo la CC BY-NC-ND licencia (http://creativecommons. org/licencias/by-nc-nd/4.0/).

Citar como/Cite as: Martínez-Gómez, J. A., Bolívar-Suárez, Y., Rey-Anacona, C. A., Ramírez-Ortiz, L. C., Lizarazo-Ojeda, A. M. y Yanez-Perúnununi, L. Y. (2021). Esquemas Tradicionales de Roles Sexuales de Género, Poder en las Relaciones y Violencia en el Noviazgo. Revista lberoamericano de Psicologia y Salud, 12(1), 1-16. Doi: hitps://doi.org/10.23923/i.rips.2021.01.041 reforzados o castigados en fusión de ser hombre o mujer; lo que facilitaría la manifestación de un conjunto de actitudes y conductas basadas en esa una única visión del género como categoría (Reyes, Foshee, Niolon, Reidy, y Hall, 2016), en particular, los roles tradicionales de género que han sido entendidos como atribuciones sobre los comportamientos y responsabilidades asignadas a los individuos en función de su sexo (Bringas-Molleda et al., 2017; García-Cueto et al., 2015). 
García-Cueto et al. (2015) señalan que existen actitudes sexistas y actitudes igualitarias o trascendentes hacia los roles de género, las primeras, se centran en una visión tradicional de mantener las desigualdades por sexo, imponiendo conductas y cogniciones a hombres y mujeres en el ámbito familiar, laboral y social, basadas en las creencias, que han estado sustentadas en el género, lo que delimitan el alcance en el desempeño de funciones acordes a ser hombre o mujer; las segundas, se enfocan en entender los comportamientos y pensamientos de los individuos en forma indiferenciada, sin adjudicar aspectos propios a cada género, es decir, este tipo de actitudes propenden por la igualdad, evitando la asignación de tareas o roles según el sexo.

Los roles de género (actitudes sexistas y actitudes igualitarias o trascendentes) se han relacionado con las conductas de violencia en las relaciones de pareja en la adolescencia (García-Díaz et al., 2018), en los últimos años la violencia en el noviazgo se ha considerado como un problema de salud pública, por su impacto negativo en la salud física y mental de víctimas y victimarios (Wincentak, Conolly, y Card, 2017; Peña et al., 2018; Cortés-Ayala et al.,2015).

Los estudios de los roles de género y la violencia en el noviazgo han demostrado que existe una relación entre las actitudes sexistas y actitudes igualitarias, con la capacidad que tienen los miembros de la pareja para identificar y reconocer cuando es víctima de maltrato en las relaciones de parejas jóvenes, por ejemplo, García-Díaz et al. (2018) desarrollaron un estudio en 57 centros de educación secundaria, profesional y universitaria de cinco provincias españolas con la participación de 4.337 estudiantes de entre 15 y 26 años, los resultados del estudio muestran que tener actitudes de género igualitarias o trascendentes favorecen la identificación de las experiencias de maltrato en las relaciones de pareja. Específicamente las actitudes trascendentes dentro del ámbito laboral se encuentran implicadas en el reconocimiento de los malos tratos en las relaciones jóvenes. Los autores concluyen que las actitudes de género influyen en la percepción que tienen los miembros de la pareja para identificar que es víctima o victimario en su relación.
De igual forma, Bringas-Molleda et al. (2017) desarrollaron una investigación con 2013 participantes mexicanos y argentinos, encontraron que quienes muestran actitudes orientadas hacia la igualdad de género informan mayores niveles de victimización, mientras que quienes presentan más actitudes sustentadas en el sexismo tienden a referir menores índices de victimización. Según los autores, esto podría estar vinculado a la capacidad para reconocer las diversas formas de abuso en el contexto de una relación de pareja, ya que la normalización de conductas violentas dificulta el reconocimiento de los malos tratos en el noviazgo.

De igual forma, ha sido estudiado en el contexto de las relaciones de pareja el grado en que una persona aprueba estándares sociales divergentes para hombres y mujeres en lo que se refiere a la sexualidad, denominado como doble estándar sexual (Emmerink, 2017), encontrándose que los noviazgos entre adultos jóvenes siguen guiones sexuales basados en el género, en los cuales se espera que las mujeres deben ser sumisas y los hombres dominantes en lo que respecta a las relaciones sexuales (Eaton y Rose, 2011 ; Sanchez, Fetterolf, y Rudman, 2012). Tal situación resulta de interés debido a que se ha encontrado que las mujeres que respaldan un doble estándar sexual presentan una menor autonomía y asertividad sexual, así como un mayor riesgo de involucrarse en relaciones sexuales sin protección (Danube et al., 2016; Kim et al., 2019). En los hombres, el doble estándar sexual se ha asociado con actitudes a favor de la violencia, con mayor probabilidad de ejercer algún tipo de agresión sexual (Moyano, Monge, y Sierra, 2017). Un estudio realizado en Corea del Sur con una muestra de 322 estudiantes universitarios, con edades entre los 18 y 20 años, encontró que una menor aceptación o adherencia al doble estándar sexual aumenta la capacidad para identificar violencia de tipo sexual en el noviazgo (Kim et al., 2019; Garrido-Macías, Valor-Segura, y Expósito, 2020).

Germain (2001) sostiene que el deseo de controlar y ejercer poder sobre la pareja puede resultar en actos de violencia, donde la víctima experimenta sentimientos de impotencia 
producto de las agresiones. Al estudiar las motivaciones de quienes ejercen violencia en sus relaciones de pareja, se ha encontrado que tener el control es una de las principales razones por las que una persona agrede a su pareja (Johnson y Ferraro, 2000).

Diferentes estudios han investigado el poder en las relaciones sexuales en el contexto de la violencia de pareja, donde esta variable se han asociado de forma inversa con la victimización y directa con la perpetración (Pulerwitz, Gortmaker, y Dejong, 2000; Ulibarri et al., 2014). Por ejemplo, Pulerwitz, Mathur y Woznica (2018) encontraron una asociación consistente entre el bajo poder en la relación y las experiencias de violencia física y psicológica sufridas por mujeres adolescentes y jóvenes con edades entre los 15 y 24 años. De igual forma, los hallazgos de investigaciones como la de Filson et al. (2009) han revelado que las mujeres que se sienten sin poder en sus relaciones presentan mayores puntajes de victimización de violencia de pareja, así como mayores niveles de depresión, donde el poder cumple un rol mediador.

Dado que los roles de género, en especial el doble estándar sexual con una menor autonomía y asertividad sexual en mujeres y el poder en la relaciones sexuales, se han relacionado con el maltrato en las relaciones de pareja, se diseñó un estudio que tuvo dos objetivos: primero, evaluar la relación entre el doble estándar sexual, el poder en las relaciones sexuales, las actitudes hacia los roles de género y la violencia en el noviazgo; y segundo, hacer una comparación entre una muestra de adolescentes/jóvenes colombianos y mexicanos, mediante un estudio observacional-analítico transversal con un diseño correlacional-comparativo.

\section{MÉTODO}

\section{- PARTICIPANTES}

En esta investigación el muestreo fue no probabilístico con la participaron 2,698 jóvenes y adultos $(42,9 \%$ hombres y $57,1 \%$ mujeres) con edades entre los 14 y 47 años $(M=20,15 ; D T=2,73)$ pertenecientes a los países de Colombia $(59,6 \%)$ y México $(40,4 \%)$; de los cuales corresponden 1607 colombianos
(47,5\% hombres y $52,5 \%$ mujeres) cuyas edades se encontraban entre los 14 y 41 años, y 1091 mexicanos (36,1\% hombres y $63,9 \%$ mujeres) con edades que oscilaban entre los 18 y 47 años. El 47,9\% (1291) de ellos reportaron tener una relación afectiva al momento de contestar la encuesta.

\section{-INSTRUMENTOS DE MEDIDA}

\section{FICHA SOCIODEMOGRÁFICA. Esta ficha} se creó expresamente para esta investigación, constando de preguntas sobre el género, edad, estado civil, nivel económico y nivel de estudios.

ESCALA DE DOBLE MORAL (EDM; Caron, Davis, Halterman y Stickle, 1993). Consiste en una adaptación salvadoreña de Sierra y Gutierrez-Quintanilla (2007), diseñada para evaluar el grado de aceptación de una moral más permisiva a la hora de juzgar la conducta sexual de los varones que la de las mujeres. Está compuesta por 10 ítems con cinco alternativas de respuesta, que conforman un único factor. Los autores de la adaptación salvadoreña obtuvieron una consistencia interna de ,73 en un primer estudio, demostrando en una segunda muestra de 1.349 estudiantes la estabilidad de la estructura, con un alfa de Cronbach de 0,78. Con los participantes en este estudio la escala mostró una alta consistencia interna, $\alpha=, 809$.

\section{CUESTIONARIO DE VIOLENCIA ENTRE} NOVIOS-REVISADO (DVQ-R; Rodríguez-Díaz et al., 2017). Este cuestionario es una versión reducida del Cuestionario de Violencia entre Novios - CUVINO (Rodríguez-Franco et al., 2010). Está diseñado para adolescentes que están en una relación de noviazgo o han mantenido una relación de noviazgo en los últimos seis meses y cuya duración fue de mínimo un mes. El DVQ-R está compuesto por 20 ítems, utiliza una escala tipo Likert con cinco opciones de respuesta desde 0 (Nunca) a 4 (Siempre) y su administración puede ser de forma Individual/grupal con una duración de cinco a diez minutos. El DVQ-R evalúa cinco dimensiones de la violencia en el noviazgo: (a)Violencia por coerción: Comportamientos explícitos dirigidos a ejercer presión sobre la 
pareja para forzar su voluntad o su conducta, los ítems que lo conforman son: 1, 9, 25 y 38; (b) Violencia sexual: Comportamientos sexistassexuales, como juegos sexuales no deseados por la pareja, así como sentirse obligado a realizar determinados actos y tocamientos sexuales. Los ítems que lo conforman son: 2, 10, 26 y 39; (c) Violencia física: Golpes, empujones, heridas o de forma indirecta, a través del daño a objetos con significación emocional para la víctima. Los ítems que lo conforman son: 5, 13, 20 y 21; (d) Violencia por desapego: Comportamientos relacionados con una actitud de indiferencia y descortesía hacia la pareja y sus sentimientos. Los ítems que lo conforman son: 6, 14, 30 y 32; (e) Violencia por humillación: Comportamientos de críticas personales dirigidas contra la autoestima y orgullo personal de la pareja, dejadez y denegación de apoyo y conductas tendentes a rebajar la estimación de una persona. Los ítems que lo conforman son: 15, 23, 40 y 41.

Su validación se desarrolló con una muestra de 6.138 hombres y mujeres adolescentes y jóvenes (con edades entre 15 y 26 años) españoles. La consistencia interna para las cinco escalas osciló entre ,64 y ,74. Para estimar la validez del instrumento se utilizó el Análisis Factorial Confirmatorio y los resultados de los parámetros del modelo final fueron $(N=6,138)$; $\mathrm{TLI}=0,94 ; \mathrm{CFI}=0,95 ; \mathrm{RMSEA}=0,018,95 \%$ C.I. $=0,016,0,020$.

\section{ESCALA DE PODER EN LAS RELACIONES} SEXUALES (Pulerwitz, Gortmaker, y Dejong, 2000). Esta escala consiste en 23 ítems que abordan el poder en las relaciones íntimas y sexuales. Consta de dos sub-escalas que miden el control en las relaciones ( 15 ítems) y el dominio en la toma de decisiones (8 ítems). Los autores del instrumento estudiaron su consistencia interna encontrando que a nivel general esta es de ,84, y por subescalas de ,86 y, 62 respectivamente. Desde su desarrollo, este instrumento se ha utilizado en numerosos estudios que exploran el poder en la relación como un determinante del riesgo sexual dentro de las relaciones primarias.

ESCALA DE ACTITUDES DE ROL DE GÉNERO (García-Cueto et al., 2015). Este instrumento se desarrolló como una escala mejorada para la evaluación de las actitudes de rol de género con base en la perspectiva teórica de la igualdad de género, en una muestra de 2,136 jóvenes españoles. Consiste en 20 enunciados que reflejan las actitudes del individuo frente a los roles de género que se deben desempeñar en la sociedad, tanto desde un punto de vista igualitario (e.g., "las personas deben ser tratadas de igual forma sin importar su sexo"), como desde un punto de vista basado en las funciones sociales (e.g., "las madres deberían tomar la mayor parte de las decisiones sobre la crianza de sus hijos"). De acuerdo con esto, el instrumento mide un factor bipolar (actitudes trascendentes vs. actitudes sexistas), en áreas como la familia, las relaciones sociales y el empleo. Por otra parte, consta de cinco opciones de respuesta que van desde 1 (Totalmente de acuerdo) hasta 5 (Totalmente en desacuerdo). La escala mostró una alta consistencia interna, 99.

\section{-PROCEDIMIENTO}

Se contactó con las directivas de las instituciones educativas para solicitar la autorización de ingreso para administrar, de forma colectiva, los cuestionarios a los participantes en los salones de clase. Al ingresar a los salones se suministró la siguiente información: a) el procedimiento de la investigación y sus posibles molestias; b) la posibilidad de no aceptar participar en el estudio o de retirarse durante la aplicación de los instrumentos, sin que ello conllevase repercusiones académicas o de otro tipo; c) el anonimato y el mantenimiento reservado de los cuestionarios. Después se solicitó la firma del formato de consentimiento informado de las personas que manifestaban la intención de participar voluntariamente en la investigación. Para el caso en el que el participante fuera menor de edad, este debía contar con la autorización de sus padres o tutor responsable, por lo tanto, el consentimiento informado debía ser firmado por estos y el menor.

\section{- CONSIDERACIONES ÉTICAS}

Este proyecto fue avalado por el comité de Bioética de la Universidad de Sonora- México. 
Dentro de las consideraciones se cumplió con lo establecido en la resolución 008430 de 1993 del Ministerio de Salud de Colombia, ley 1090 del 2006 (manual deontológico y bioético de la psicología), Ley 911 de 2006 (código deontológico enfermería) y Ley 1581 de 2012 (protección de datos personales).

\section{-ANÁLISIS DE DATOS}

Para alcanzar los objetivos de esta investigación se emplearon: a) análisis descriptivos a partir de las medidas de dispersión, tendencia central y frecuencia; b) análisis de correlaciones para identificar la relación de la violencia en el noviazgo con las actitudes hacia los roles de género, el doble estándar sexual y el poder en las relaciones sexuales; y c) análisis de diferencias de medias mediante el estadístico t de Student para grupos independientes cuando el factor de agrupamiento tenía dos niveles, y un ANOVA $2 X^{2}$ para la comparación de medias entre dos factores con dos niveles cada uno. Los tamaños del efecto se obtuvieron mediante la $d$ de
Cohen para la comparación de dos medias y para la estimación de la magnitud con la probabilidad de superioridad del tamaño del efecto, que informa en probabilidades de la superioridad del tamaño del efecto (PSTE) observado sobre el total de los posibles (Redondo, Fariña, Seijo, Novo, y Arce, 2019).

\section{RESULTADOS}

Referente a los análisis de relación entre la violencia en el noviazgo y las actitudes hacia los roles de género, doble estándar sexual y poder en las relaciones sexuales en los participantes de la muestra de Colombia efectuados a través del coeficiente de correlación de Pearson (ver Tabla $1)$, se encontró que las actitudes trascendentes se correlacionaron de forma negativa y débil con la violencia física, sexual y humillación ejercidas. Asimismo, las actitudes sexistas se correlacionaron directa y significativamente, aunque de manera débil con la violencia física, sexual, humillación y coerción recibidas, así como con la violencia sexual, humillación, desapego y coerción ejercidas.

Tabla 1

Correlaciones entre la violencia en el noviazgo y las actitudes hacia los roles de género, el doble estándar sexual y el poder en las relaciones sexuales (Colombia)

\begin{tabular}{|c|c|c|c|c|c|c|}
\hline$N=1518$ & $\begin{array}{c}\text { Actitudes } \\
\text { transcendentes }\end{array}$ & $\begin{array}{l}\text { Actitudes } \\
\text { sexistas }\end{array}$ & $\begin{array}{l}\text { Aceptación } \\
\text { del doble } \\
\text { estándar } \\
\text { sexual }\end{array}$ & $\begin{array}{l}\text { Control } \\
\text { de la } \\
\text { Relación }\end{array}$ & $\begin{array}{c}\text { Dominio } \\
\text { en la } \\
\text { Toma de } \\
\text { Decisiones }\end{array}$ & $\begin{array}{c}\text { Poder en las } \\
\text { Relaciones } \\
\text { Sexuales }\end{array}$ \\
\hline Violencia Física recibida &,- 041 &, $111^{\star \star \star}$ &, $139 * \star *$ &, $260 * \star \star$ &,$- 100^{\star * \star}$ &, $238 * \star \star$ \\
\hline Violencia Sexual recibida &,- 011 &, $081^{* \star}$ &, $093^{* * \star}$ &, $247^{\star \star \star}$ &,$- 136 * * *$ &, $218^{\star * *}$ \\
\hline Humillación recibida &,- 025 &, $082^{\star *}$ &, $080 * \star$ &, $293 * \star \star$ &,$- 154 * \star \star$ &, $260 * * *$ \\
\hline Desapego recibida &, 025 & ,024 &, 036 &, $241^{\star * \star}$ &,$- 090^{\star \star \star}$ & 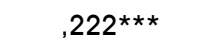 \\
\hline Coerción recibida &,- 018 &, $107 * \star \star$ &, $119 * \star \star$ &, $329 * * *$ &,- 044 &, $319 * \star \star$ \\
\hline Violencia Física ejercida &,$- 059 *$ & ,046 &, $052 *$ &, $169 * * *$ &, 028 &, $175^{\star \star \star}$ \\
\hline Violencia Sexual ejercida &,$- 104^{\star * \star}$ &, $210 * \star *$ &, $188^{* * *}$ &, $225^{\star \star *}$ &, 043 &, $234^{\star * *}$ \\
\hline Humillación ejercida &,$- 054^{*}$ &, $172^{\star \star \star}$ &, $149 * * \star$ &, $208 * \star \star$ & ,037 &, $216 * \star \star$ \\
\hline Desapego ejercida &,- 025 &, $114^{\star \star \star}$ &, $120 * \star \star$ &, $164^{\star \star \star}$ &, $055^{\star}$ &, $175^{\star \star \star}$ \\
\hline Coerción ejercida &,- 043 &, $132 * \star \star$ &, $175^{\star \star \star}$ &, $296 * \star \star$ & 017 &, $299 * * \star$ \\
\hline
\end{tabular}


La aceptación del doble estándar sexual se correlacionó positivamente de forma significativa y débil con la violencia física, sexual, humillación, desapego, coerción recibidas y ejercidas excepto el desapego recibido. En contraste, el dominio en la toma de decisiones se correlacionó negativamente y de manera significativa y débil con la violencia física, sexual humillación y desapego recibidas, a diferencia del desapego ejercido que se correlacionó directa y débil con el dominio en la toma de decisiones en jóvenes y adultos colombianos.

Con relación al control de la relación y el poder en las relaciones sexuales, todas las variables analizadas de violencia ejercida y recibida se correlacionaron positivamente y de forma significativa y de manera débil en jóvenes y adultos de Colombia.

El análisis de relación entre la violencia en el noviazgo y las actitudes hacia los roles de género, doble estándar sexual y poder en las relaciones sexuales en la muestra de México realizado a partir del coeficiente de correlación de Pearson (ver Tabla 2) se observa que las actitudes trascendentes se correlacionaron negativamente y de forma significativa con la violencia física recibida y la violencia sexual ejercida. Además, las actitudes sexistas y la aceptación del doble estándar sexual se correlacionaron directa y significativamente débil con todas las variables de violencia recibida y ejercida a excepción de la humillación recibida.

El dominio en la toma de decisiones en jóvenes y adultos mexicanos correlacionó negativa y forma significativa, aunque débil con la violencia sexual, humillación desapego y coerción recibida. Sin embargo, con respecto a la violencia física, sexual, humillación ejercidas estas correlacionaron positivamente de manera significativa y débil con el dominio en la toma de decisiones de jóvenes y adultos de México. Cabe destacar que al igual que en los jóvenes y adultos colombianos, el control y el poder en las relaciones sexuales se correlacionaron directa, positiva y débil en todas las variables de violencia ejercida y recibida en participantes mexicanos.

\section{Tabla 2}

Correlaciones entre la violencia en el noviazgo y las actitudes hacia los roles de género, el doble estándar sexual y el poder en las relaciones sexuales (México)

\begin{tabular}{|c|c|c|c|c|c|c|}
\hline$N=950$ & $\begin{array}{c}\text { Actitudes } \\
\text { transcendentes }\end{array}$ & $\begin{array}{l}\text { Actitudes } \\
\text { sexistas }\end{array}$ & $\begin{array}{l}\text { Aceptación } \\
\text { del doble } \\
\text { estándar } \\
\text { sexual }\end{array}$ & $\begin{array}{l}\text { Control } \\
\text { de la } \\
\text { Relación }\end{array}$ & $\begin{array}{c}\text { Dominio } \\
\text { en la } \\
\text { Toma de } \\
\text { Decisiones }\end{array}$ & $\begin{array}{c}\text { Poder en las } \\
\text { Relaciones } \\
\text { Sexuales }\end{array}$ \\
\hline Violencia Física recibida &,$- 081^{*}$ &, $154^{\star \star *}$ &, $164^{\star * *}$ &, $249 * * *$ &,- 045 &, $239 * * *$ \\
\hline Violencia Sexual recibida &,- 023 &, $064^{\star}$ &, $072^{*}$ &, $226 * \star *$ &,$- 161^{* * *}$ &, $193^{* * *}$ \\
\hline Humillación recibida &,- 018 &, 060 &, 029 &, $250 * \star \star$ &,$- 192 * \star \star$ &, $210 * \star \star$ \\
\hline Desapego recibida &, 020 &, $082^{\star}$ &, $075^{*}$ &, $230 * * *$ &,$- 162^{\star * *}$ &, $196 * * *$ \\
\hline Coerción recibida &,- 035 &, $115^{\star \star *}$ &, $117 * * *$ &, $281 * \star *$ &,$- 149 * * *$ &, $249 * \star *$ \\
\hline Violencia Física ejercida &,- 059 &, $128 * \star *$ &, $136 * \star *$ &, $242^{\star \star \star}$ &, $076^{*}$ &, $258 * \star *$ \\
\hline Violencia Sexual ejercida &,$- 127^{\star * \star}$ &, $177^{\star * *}$ &, $188^{* * *}$ &, $189 * * *$ &, $122 * \star *$ &, $214^{\star * *}$ \\
\hline Humillación ejercida &,- 055 &, $118^{* * *}$ &, $127^{\star * *}$ &, $177^{\star * *}$ &, $073^{*}$ &, $192 * \star \star$ \\
\hline Desapego ejercida &,- 056 &, $206 * \star \star *$ &, $205^{\star * *}$ &, $181^{\star * *}$ & 012 &, $183^{\star \star \star}$ \\
\hline Coerción ejercida &,- 043 &, $113^{* \star *}$ &, $116^{* * *}$ &, $230 * \star *$ &, $064^{*}$ &, $242^{* * *}$ \\
\hline
\end{tabular}


En cuanto al análisis de diferencias de violencia en el noviazgo y las actitudes hacia los roles de género, doble estándar sexual y poder en las relaciones sexuales en Colombia y México, llevado a cabo por el estadístico de t de student, se encontraron diferencias estadísticamente significativas entre la violencia física, desapego y coerción sufridas, violencia física, humillación, desapego y coerción ejercidas, así como la perpetración, victimización, actitudes trascendentes, aceptación del doble estándar sexual, control en la relación, dominio en la toma de decisiones y poder en las relaciones sexuales en México y Colombia. En ese sentido, se observó que las medias de la violencia física sufrida y ejercida, las actitudes trascendentes, la aceptación del doble estándar sexual, el control de la relación y el poder en las relaciones sexuales fueron mayores en los participantes mexicanos. Mientras que, en las modalidades de desapego, coerción, sufrida, violencia sexual, humillación, desapego, coerción ejercida, perpetración, victimización y dominio en la toma de decisiones la media fue mayor en jóvenes y adultos colombianos (ver Tabla 3). Respecto a las variables de violencia sexual sufrida, ejercida y las actitudes sexistas no se encontraron diferencias significativas.

\section{Tabla 3}

Diferencias por país († Student) de la violencia en el noviazgo, las actitudes hacia los roles de género, el doble estándar sexual y el poder en las relaciones sexuales.

\begin{tabular}{|c|c|c|c|c|c|c|}
\hline Variable & País & $N$ & $M$ & $D T$ & $t$ & $d$ \\
\hline \multirow{2}{*}{ Violencia Física Recibida } & Colombia & 1518 & 0,46 & 1,14 & \multirow{2}{*}{$-2,07^{\star}$} & \multirow{2}{*}{0,08} \\
\hline & México & 950 & 0,57 & 1,25 & & \\
\hline \multirow{2}{*}{ Violencia Sexual Recibida } & Colombia & 1518 & 0,62 & 1,58 & \multirow{2}{*}{$-0,74$} & \multirow{2}{*}{0,03} \\
\hline & México & 950 & 0,66 & 1,64 & & \\
\hline \multirow{2}{*}{ Violencia Humillación Recibida } & Colombia & 1518 & 1,30 & 1,85 & \multirow{2}{*}{1,70} & \multirow{2}{*}{0,07} \\
\hline & México & 950 & 1,17 & 1,91 & & \\
\hline \multirow{2}{*}{ Violencia Desapego Recibida } & Colombia & 1518 & 2,91 & 2,81 & \multirow{2}{*}{$4,03 * \star *$} & \multirow{2}{*}{0,18} \\
\hline & México & 950 & 2,43 & 3,02 & & \\
\hline \multirow{2}{*}{ Violencia Coerción Recibida } & Colombia & 1518 & 2,65 & 2,67 & \multirow{2}{*}{$3,20^{\star *}$} & \multirow{2}{*}{0,16} \\
\hline & México & 950 & 2,28 & 2,98 & & \\
\hline \multirow{2}{*}{ Violencia Física Ejercida } & Colombia & 1518 & 0,34 & ,957 & \multirow{2}{*}{$-4,47 * * \star$} & \multirow{2}{*}{0,18} \\
\hline & México & 950 & 0,54 & 1,17 & & \\
\hline \multirow{2}{*}{ Violencia Sexual Ejercida } & Colombia & 1518 & 0,33 & 0,99 & \multirow{2}{*}{1,525} & \multirow{2}{*}{0,06} \\
\hline & México & 950 & 0,27 & 0,93 & & \\
\hline \multirow{2}{*}{ Violencia Humillación Ejercida } & Colombia & 1518 & 1,09 & 1,47 & \multirow{2}{*}{ 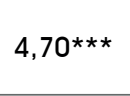 } & \multirow{2}{*}{0,19} \\
\hline & México & 950 & 0,84 & 1,23 & & \\
\hline \multirow{2}{*}{ Violencia Desapego Ejercida } & Colombia & 1518 & 2,76 & 2,49 & \multirow{2}{*}{$10,05^{\star \star \star}$} & \multirow{2}{*}{0,40} \\
\hline & México & 950 & 1,84 & 2,04 & & \\
\hline \multirow{2}{*}{ Violencia Coerción Ejercida } & Colombia & 1518 & 1,97 & 1,92 & \multirow{2}{*}{$4,06 * \star \star *$} & 016 \\
\hline & México & 950 & 1,64 & 2,02 & & 0,10 \\
\hline Actitudes transcendentes & Colombia & 1607 & 24,5 & 4,24 & $-270 * *$ & 10 0 1 \\
\hline Acilludes iramscendentes & México & 1091 & 25,0 & 4,12 & $-2, / 0$ & 0,10 \\
\hline Actitudec ceyictas & Colombia & 1607 & 32,2 & 7,68 & 057 & ?ח \\
\hline MCilinues sexisids & México & 1091 & 32,0 & 8,42 & 0,0 & 0,02 \\
\hline Acentación del doble están dar sexual & Colombia & 1607 & 20,1 & 6,39 & $-270 * *$ & 010 10 \\
\hline " & México & 1091 & 20,8 & 5,93 & $-2,1>$ & 0,10 \\
\hline Control de la Pelación & Colombia & 1607 & 64,8 & 8,32 & $004 * \star *$ & 0.40 \\
\hline 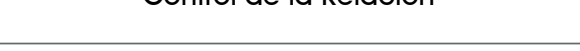 & México & 950 & 68,3 & 8,68 & $-9,94$ & 0,40 \\
\hline Dominin on la Toma de Decisiones & Colombia & 1607 & 16,3 & 1,73 & $150 * * *$ & $018 \quad 7 \quad \rightarrow$ \\
\hline 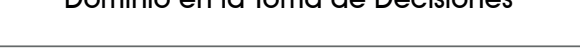 & México & 950 & 15,9 & 1,80 & $4,54^{n}$ & 0,18 \\
\hline Poder en las relaciones sexuales (suma de & Colombia & 1607 & 81,1 & 8,35 & $807 * \star * *$ & $036>0$ \\
\hline control y dominancia) & México & 950 & 84,20 & 8,70 & $-0,91$ r & 0,00 \\
\hline
\end{tabular}


Ejecutado un ANOVA con un diseño 2(sexo: mujer vs. hombre) X 2(País: México vs. Colombia), los resultados mostraron diferencias estadísticamente significativas entre grupos con respecto a la victimización en el noviazgo (ver Tabla 4). Por su parte, los contrastes a posteriori, con el nivel de protección de Bonferroni, informaron que, para el caso de la violencia física recibida, los hombres colombianos y mexicanos presentaron puntajes significativamente mayores de violencia física que las mujeres colombianas $(d=0,31[$ PSTE $=, 174])$ y mexicanas $(d=$ $0,29[$ PSTE $=, 166])$, respectivamente. Respecto a la violencia sexual recibida, las mujeres mexicanas tuvieron puntajes significativamente mayores que los hombres mexicanos $(d=$ $0,18[$ PSTE $=$, 103])
En el caso de la violencia por humillación recibida, los hombres colombianos presentaron puntajes mayores que las mujeres colombianas $(d=0,18[$ PSTE $=, 103])$ y mexicanas $(d=$ $0,19[$ PSTE $=, 103])$. En cuanto a la violencia por desapego, los hombres y las mujeres colombianas presentaron mayores puntajes que los hombres mexicanos $(d=0,31$ [PSTE $=$ ,174] y $0,24[$ PSTE $=, 135]$, respectivamente). Finalmente, de la violencia por coerción se encontró que los hombres colombianos presentaron mayores puntajes que las mujeres colombianas $(d=0,30[$ PSTE $=, 166])$ y que los hombres y las mujeres mexicanas $(d=$ $0,27[$ PSTE $=, 151]$ y $0,28[$ PSTE $=, 151]$, respectivamente).

Tabla 4

Diferencias entre los tipos de violencia recibida por hombres y mujeres en una muestra de jóvenes colombianos y mexicanos $(N=2698)$

\begin{tabular}{|c|c|c|c|c|}
\hline Violencia Física Recibida & $M(D T)$ & $g l$ & $F$ & $p$ \\
\hline Hombres Colombia & $0,65(1,31)$ & \multirow{4}{*}{3} & \multirow{4}{*}{19,03} & \multirow{4}{*}{, 000} \\
\hline Mujeres Colombia & $0,30(0,94)$ & & & \\
\hline Hombres México & $0,80(1,59)$ & & & \\
\hline Mujeres México & $0,44(1,00)$ & & & \\
\hline \multicolumn{5}{|l|}{ Violencia Sexual Recibida } \\
\hline Hombres Colombia & $0,67(1,58)$ & \multirow{4}{*}{3} & \multirow{4}{*}{3,36} & \multirow{4}{*}{,018 } \\
\hline Mujeres Colombia & $0,57(1,58)$ & & & \\
\hline Hombres México & $0,47(1,38)$ & & & \\
\hline Mujeres México & $0,77(1,75)$ & & & \\
\hline \multicolumn{5}{|c|}{ Violencia por Humillación Recibida } \\
\hline Hombres Colombia & $1,48(1,92)$ & \multirow{4}{*}{3} & \multirow{4}{*}{4,94} & \multirow{4}{*}{, 002} \\
\hline Mujeres Colombia & $1,15(1,78)$ & & & \\
\hline Hombres México & $1,21(1,76)$ & & & \\
\hline Mujeres México & $1,15(1,99)$ & & & \\
\hline \multicolumn{5}{|c|}{ Violencia por Desapego Recibida } \\
\hline Hombres Colombia & $2,98(2,67)$ & \multirow{4}{*}{3} & \multirow{4}{*}{7,34} & \multirow{4}{*}{, 000} \\
\hline Mujeres Colombia & $2,85(2,93)$ & & & \\
\hline Hombres México & $2,15(2,75)$ & & & \\
\hline Mujeres México & $2,58(3,16)$ & & & \\
\hline \multicolumn{5}{|c|}{ Violencia por Coerción Recibida } \\
\hline Hombres Colombia & $3,07(2,70)$ & \multirow{4}{*}{3} & \multirow{4}{*}{7,77} & \multirow{4}{*}{, 000} \\
\hline Mujeres Colombia & $2,28(2,60)$ & & & \\
\hline Hombres México & $2,33(2,79)$ & & & \\
\hline Mujeres México & $2,25(3,08)$ & & & \\
\hline \multicolumn{5}{|l|}{ Nota. $g l=$ Grados de libertad. } \\
\hline
\end{tabular}


Respecto a la violencia ejercida, se ejecutó de nuevo un ANOVA con un diseño 2 (sexo: mujer vs. hombre) X 2(País: México vs. Colombia), el cual reveló diferencias estadísticamente significativas entre los grupos para esta variable (ver Tabla 5). Concretamente, los contrastes a posteriori, con el nivel de protección de Bonferroni, informaron que, en la violencia física, los hombres mexicanos y las mujeres mexicanas presentan puntajes significativamente mayor que los hombres colombianos $(d=0,17$ [PSTE $=$ ,096] y 0,26[PSTE $=$,143], respectivamente). Por su parte, las mujeres mexicanas tuvieron mayores puntajes que las mujeres colombianas $(d=0,20[$ PSTE $=, 1111])$. En la violencia sexual, los hombres colombianos presentaron mayores puntajes que las mujeres colombianas $(d=$ $0,41[$ PSTE $=, 228]$ ) y mexicanas ( $d=0,39$ [PSTE $=, 221]$ ). Asimismo, también los hombres mexicanos presentaron mayores puntajes que las mujeres, tanto colombianas ( $d=0,32$ [PSTE $=, 182])$ como mexicanas $(d=0,31$ [PSTE $=$ ,174]). Respecto a la violencia por humillación, los hombres colombianos presentaron mayores puntajes que las mujeres colombianas $(d=$ 0,32 [PSTE $=$, 182]), así como que los hombres mexicanos $(d=0,25[$ PSTE $=, 143])$ y las mujeres mexicanas $(d=0,38[P S T E=, 213])$. En la violencia por desapego, los hombres colombianos presentaron mayores puntuaciones que las mujeres colombianas $(d=0,28$ [PSTE $=$ ,151]). Por último, en cuanto a la violencia por

Tabla 5

Diferencias entre los tipos de violencia ejercida por hombres y mujeres entre una muestra de jóvenes colombianos y mexicanos $(N=2698)$

\begin{tabular}{|c|c|c|c|c|}
\hline Violencia Física Recibida & $M(D T)$ & $g l$ & $F^{a}$ & $p^{b}$ \\
\hline Hombres Colombia & $0,31(0,90)$ & \multirow{4}{*}{3} & \multirow{4}{*}{7,775} & \multirow{4}{*}{, 000} \\
\hline Mujeres Colombia & $0,36(1,00)$ & & & \\
\hline Hombres México & $0,50(1,38)$ & & & \\
\hline Mujeres México & $0,56(1,04)$ & & & \\
\hline \multicolumn{5}{|l|}{ Violencia Sexual Ejercida } \\
\hline Hombres Colombia & $0,54(1,22)$ & \multirow{4}{*}{3} & \multirow{4}{*}{28,122} & \multirow{4}{*}{,000 } \\
\hline Mujeres Colombia & $0,15(0,67)$ & & & \\
\hline Hombres México & $0,45(1,33)$ & & & \\
\hline Mujeres México & $0,16(0,59)$ & & & \\
\hline \multicolumn{5}{|c|}{ Violencia por Humillación Ejercida } \\
\hline Hombres Colombia & $1,34(1,67)$ & \multirow{4}{*}{3} & \multirow{4}{*}{23,137} & \multirow{4}{*}{, 000} \\
\hline Mujeres Colombia & $0,88(1,22)$ & & & \\
\hline Hombres México & $0,96(1,26)$ & & & \\
\hline Mujeres México & $0,78(1,21)$ & & & \\
\hline \multicolumn{5}{|c|}{ Violencia por Desapego Ejercida } \\
\hline Hombres Colombia & $3,14(2,63)$ & \multirow{4}{*}{3} & \multirow{4}{*}{43,357} & \multirow{4}{*}{,000 } \\
\hline Mujeres Colombia & $2,44(2,31)$ & & & \\
\hline Hombres México & $1,97(2,11)$ & & & \\
\hline Mujeres México & $1,77(1,99)$ & & & \\
\hline \multicolumn{5}{|c|}{ Violencia por Coerción Ejercida } \\
\hline Hombres Colombia & $2,20(1,98)$ & \multirow{4}{*}{3} & \multirow{4}{*}{12,872} & \multirow{4}{*}{, 000} \\
\hline Mujeres Colombia & $1,76(1,85)$ & & & \\
\hline Hombres México & $1,49(1,88)$ & & & \\
\hline Mujeres México & $1,72(2,10)$ & & & \\
\hline
\end{tabular}


coerción, los hombres colombianos presentan mayores puntajes en este tipo de violencia con respecto a las mujeres colombianas $(d=$ $0,23[$ PSTE $=, 127]$ ), así como a los hombres mexicanos $(d=0,37[P S T E=, 205])$ y mujeres mexicanas $(d=0,24[$ PSTE $=, 135])$.

Finalmente, se ejecutó un ANOVA con un diseño 2(sexo: mujer vs. hombre) X 2(País: México vs. Colombia) para analizar las actitudes hacia los roles de género. Los resultados informaron de diferencias significativas entre los grupos para esta variable (ver Tabla 6). Por su parte, los contrastes a posteriori, con el nivel de protección de Bonferroni, señalan que las mujeres colombianas y mexicanas presentaron puntuaciones significativamente mayores en las actitudes trascendentes en comparación con los hombres colombianos ( $d=0,41$ [PSTE $=$ ,228]) y mexicanos ( $d=0,42[$ PSTE $=, 236])$, respectivamente. En las actitudes sexistas, los hombres mexicanos obtuvieron los puntajes más altos, difiriendo de forma significativa con los hombres colombianos ( $d=0,16[$ PSTE $=, 088]$ ) y las mujeres colombianas ( $d=0,77$ PSSTE $=, 411])$ y mexicanas $(d=0,76$ [PSTE $=$ ,411]). Asimismo, los puntajes de los hombres colombianos también fueron significativamente mayores que los de las mujeres colombianas $(d=0,61[$ PSTE $=, 323])$ y mexicanas $(d=$ $0,62[$ PSTE $=, 340])$.

\section{Tabla 6}

Diferencias entre las actitudes hacia los roles de género, el poder en las relaciones y el doble estándar sexual de hombres y mujeres entre una muestra de jóvenes colombianos y mexicanos $(N=2698)$

\begin{tabular}{|c|c|c|c|c|c|}
\hline Actitudes roles de género & \multirow{2}{*}{$M(D T)$} & \multirow{2}{*}{$g l$} & \multirow{2}{*}{$F a$} & \multirow{2}{*}{$p^{b}$} & \multirow{2}{*}{ TE } \\
\hline Actitudes Trascendentes & & & & & \\
\hline Hombres Colombia & $23,6(4,52)$ & \multirow{4}{*}{3} & \multirow{4}{*}{40,071} & \multirow{4}{*}{, 000} & \multirow{4}{*}{, 21} \\
\hline Mujeres Colombia & $25,3(3,79)$ & & & & \\
\hline Hombres México & $23,9(4,36)$ & & & & \\
\hline Mujeres México & $25,6(3,85)$ & & & & \\
\hline \multicolumn{6}{|l|}{ Actitudes Sexistas } \\
\hline Hombres Colombia & $34,5(7,91)$ & \multirow{4}{*}{3} & \multirow{4}{*}{97,914} & \multirow{4}{*}{, 000} & \multirow{4}{*}{, 36} \\
\hline Mujeres Colombia & $30,0(6,80)$ & & & & \\
\hline Hombres México & $35,8(8,88)$ & & & & \\
\hline Mujeres México & $29,8(7,32)$ & & & & \\
\hline \multicolumn{5}{|l|}{ Poder relaciones sexuales } & \\
\hline \multicolumn{6}{|l|}{ Control en la Relación } \\
\hline Hombres Colombia & $66,3(8,30)$ & \multirow{4}{*}{3} & \multirow{4}{*}{50,362} & \multirow{4}{*}{, 000} & \multirow{4}{*}{, 25} \\
\hline Mujeres Colombia & $63,4(8,09)$ & & & & \\
\hline Hombres México & $68,4(9,89)$ & & & & \\
\hline Mujeres México & $68,2(7,94)$ & & & & \\
\hline \multicolumn{6}{|c|}{ Dominancia en la Toma de Decisiones } \\
\hline Hombres Colombia & $16,2(1,73)$ & \multirow{4}{*}{3} & \multirow{4}{*}{7,163} & \multirow{4}{*}{,000 } & \multirow{4}{*}{16} \\
\hline Mujeres Colombia & $16,3(1,73)$ & & & & \\
\hline Hombres México & $16,0(1,77)$ & & & & \\
\hline Mujeres México & $15,9(1,82)$ & & & & \\
\hline \multicolumn{6}{|l|}{ Doble Estándar Sexual } \\
\hline Hombres Colombia & $22,5(6,39)$ & \multirow{4}{*}{3} & \multirow{4}{*}{119,615} & \multirow{4}{*}{, 000} & \multirow{4}{*}{, 35} \\
\hline Mujeres Colombia & $17,9(5,55)$ & & & & \\
\hline Hombres México & $23,1(6,88)$ & & & & \\
\hline Mujeres México & $19,4(4,85)$ & & & & \\
\hline \multicolumn{5}{|l|}{ Nota. $g l=$ Grados de libertad. } & \\
\hline
\end{tabular}


En relación al poder en las relaciones sexuales, los hombres mexicanos presentaron la media más alta respecto al control, difiriendo de forma significativa con los hombres colombianos $(d=0,24[$ PSTE $=, 135])$, así como con las mujeres colombianas ( $d=0,57$ [PSTE $=, 311]$ ). Asimismo, las mujeres mexicanas presentaron puntuaciones significativamente mayores que las de las mujeres colombianas ( $d=0,60$ [PSTE $=, 333])$ y que los hombres colombianos ( $d=$ $0,23[$ PSTE $=, 127]$ ). Los puntajes de los hombres colombianos también fueron significativamente mayores que los de las mujeres colombianas con respecto al control en las relaciones. Continuando con el dominio en la toma de decisiones, las mujeres colombianas tuvieron los puntajes más altos, presentando diferencias con respecto a los puntajes de las mujeres mexicanas $(d=0,23[$ PSTE $=, 127])$ y a los hombres mexicanos ( $d=0,17[\mathrm{PSTE}=, 096])$. De igual forma, los hombres colombianos presentaron puntajes significativamente mayores que los de las mujeres mexicanas $(d=0,17$ [PSTE $=$ ,1096]).

Finalmente, se encontró que los hombres mexicanos presentaron los puntajes más altos en doble estándar sexual, significativamente mayores a los obtenidos por las mujeres mexicanas $(d=0,65[$ PSTE $=, 354])$ y colombianas $(d=0,87[$ PSTE $=, 465])$. Asimismo, los hombres colombianos presentaron también puntajes significativamente mayores que las mujeres colombianas ( $d=0,82[$ PSTE $=, 438]$ ) y mexicanas ( $d=0,54[$ PSTE $=, 296])$. Entre las mujeres, las mexicanas tuvieron puntajes significativamente mayores que las colombianas $(d=0,29[$ PSTE $=, 166])$.

\section{DISCUSIÓN}

Actualmente la violencia en el noviazgo está catalogada como un fenómeno grave de salud pública, afectando la integridad física y psicológica de quienes sufren estos comportamientos en su relación. Este problema ha sido relacionado con diferentes factores desencadenantes de tipo social, individual y familiar, además, existe evidencia de la relación entre el rol de género y la violencia en parejas jóvenes Borges, Heine, Dell'Áglio (2020). Por lo tanto, el objetivo principal de esta investigación ayudó a entender el fenómeno de la violencia en el noviazgo al evaluar la relación entre el doble estándar sexual, el poder en las relaciones sexuales, las actitudes hacia los roles de género en una muestra de adolescentes/ jóvenes colombianos y mexicanos.

Al analizar la frecuencia de las conductas de violencia, se encontró que tanto hombres y mujeres mexicanos y colombianos, utilizan la violencia de forma bidireccional, estos resultados son consistentes con hallazgos de investigaciones que señalan que independiente de la condición de género se recibe y ejerce violencia en las relaciones de pareja en jóvenes, comenzando por incidentes leves que dan lugar a un proceso de escalamiento hacia eventos de mayor gravedad (Beserra et al., 2015; Oliveira et al., 2014; Martínez et al., 2016; Quesada et al., 2020). En concordancia con los hallazgos de otras investigaciones, la prevalencia de perpetración de violencia hacia la pareja es alta (Kann et al., 2018; Vivanco et al., 2015; Wincentak, Connolly. y Card, 2017) siendo la violencia por humillación, coerción y desapego las que se presentan con mayor frecuencia, esta última la más reportada con conductas como estrategias de control y manipulación basadas en la indiferencia y descortesía hacia su pareja (Cortés-Ayala et al., 2015; García et al., 2015; Martínez et al., 2016). Sin embargo, los tamaños de efecto en cada una de las tipologías de violencia, al compararlas por país y género, fueron pequeños o medianos (Álvarez-Muelas, Gómez-Berrocal, y Sierra, 2020b), por lo tanto, estas deben ser entendidas como una tendencia, no obstante, se puede justificar el uso del sexo como variable diferenciadora en el estudio de la violencia en parejas jóvenes.

Con referencia al Rol de Género, en ambos países las actitudes sexistas, caracterizadas por una percepción desigual de las funciones y responsabilidades atribuidas socialmente al hombre o a la mujer, correlacionaron de manera directa y significativa con varios tipos de violencia tanto recibida como ejercida, por lo tanto, las personas que tienen creencias y patrones culturales influenciados por su rol de género, pueden llegar a justificar o aceptar conductas 
de violencia en el marco de una relación de dominio y control, las cuales trascienden en contextos sociales, familiares y laborales (Benstead, 2020; Weber, Taylor, Cantos, Amado, y $\mathrm{O}^{\prime}$ Leary, 2019). Además, se encontró que las actitudes trascedentes correlacionan de forma negativa con algunos tipos de violencia en las relaciones de pareja, esto quiere decir que las personas que se comportan en su relación de forma igualitaria en la asignación de roles y actividades independientes del género ejercen menos conductas de violencia.

Otra variable vinculada al rol de género que se relacionó de forma significativa, en esta investigación con la violencia ejercida y recibida en el noviazgo fue en el doble estándar sexual, de esta forma, las personas que atribuyen y aceptan ciertos tipos de comportamiento sexuales apropiados socialmente para los hombres y los cuales se consideran inapropiados para las mujeres, con mayor frecuencia tienden a ejercer o ser víctima de conductas de control y violencia en su relación de pareja (Álvarez-Muelas, Gómez-Berrocal, y Sierra, 2020a; Zaikman y Marks, 2017). Al respecto la teoría del rol social reconoce que la sociedad tiene una expectativa diferencial en la conducta de hombres y mujeres, por lo tanto, el rol estará determinado por esta concepción y así se manifiesta sus pensamientos, sentimientos y comportamientos hacia los demás, luego la aprobación o no de los roles de género contribuye a la manifestación del doble estándar sexual (Eagly, Wood, y Diekman, 2000; Zaikman y Marks, 2017).

Al comparar las puntuaciones obtenidas en la escala de doble estándar sexual, por país y género, se encontró que tanto hombres mexicanos y colombianos, tienen puntuaciones superiores comparadas con las puntuaciones de las mujeres colombianas y mexicanas. Esto podría deberse que en Latinoamérica se cuenta con estructuras de género convencionales y tradicionalistas, mostrando una mayor aceptación del doble estándar sexual por parte de los hombres, quienes podrían evaluar con mayor severidad y de forma negativamente las conductas sexuales de las mujeres, cuando se salen de las estructuras tradicionales del rol de género. Investigaciones similares, donde han analizado estas variables, señalan que los hombres obtienen mayor puntuación en comparación con las mujeres respecto al doble estándar sexual (Emmerink et al., 2016; England y Bearak, 2014; Kim et al., 2019; Málaga y Rivas, 2018; Ubillos, Goiburu, Puente, y Pizarro, 2016).

No obstante, son los hombres mexicanos quienes tuvieron mayor puntuación en la escala de doble estándar sexual, esto se podría deber, a que los adolescentes mexicanos tienden a tener una concepción tradicional de las conductas y actitudes sexuales de la pareja, con una actitud favorable al doble estándar sexual, en comparación con los adolescentes colombianos. En los jóvenes mexicanos el doble estándar correlacionó positivamente con las cinco dimensiones de violencia en el noviazgo tanto ejercida como recibida. Esto nos lleva a pensar que dichas actitudes pueden justificar la perpetración de diversos actos violentos con la subsecuente aceptación por parte de las víctimas al considerar que son normales dentro del contexto socio-cultural, y como mecanismo de control por parte de victimario para someter y mantener la fidelidad de la apareja (Baumeister y Twenge, 2002). Esto se podría atribuir a que los adolescentes se encuentran inmersos en un esquema de aprendizaje social, donde a partir de la observación, imitan comportamientos que son recompensados o castigados socialmente de acuerdo con las expectativas del rol de género, haciendo que estas conductas se perpetúen en el tiempo (Bandura, 1986; Zaikman y Marks, 2017).

En este estudio se encontró que el poder en las relaciones sexuales se correlaciona de manera positiva con todas las formas de violencia en el noviazgo recibida y ejercida, donde los hombres mexicanos informan mayores conductas de control, mientras las mujeres colombianas muestran mayordominio en la toma de decisiones. De esta manera, las relaciones sexuales, podría permearse por algunas creencias culturales tradicionales, donde se genera una estructura de poder de dominancia y subordinación. En el caso específico de Latinoamérica las dinámicas sexuales en la pareja se ven afectadas por las concepciones del rol de género donde los hombres son reforzados socialmente para tener la iniciativa en la toma de decisiones de índole sexual. Esta relación ente el poder en las 
relaciones sexuales y la violencia en el noviazgo, podría deberse a que los miembros de la pareja buscan vencer al otro en la toma de decisiones y control, como resultado se tiene una relación asimétrica, por lo tanto, en el proceso de acceso a la posición de poder se puede generar conductas de violencia (Bermúdez y MeléndezRodríguez, 2020; Matsuda, McGrath, y Jallo, 2012; Sierra, Moyano, Vallejo, y Gómez, 2018).

Finalmente, los resultados de esta investigación ayudan a entender que las conductas y actitudes tradicionalista sobre el género, están relacionadas con la violencia en el noviazgo, en especial actitudes de aceptación del doble estándar sexual y poder en las relaciones. Por lo tanto, los programas de prevención e intervención con maltratadores, que se han mostrado efectivos en ciertas circunstancias (Arce, Arias, Novo, y Fariña, 2020), pueden mejorar su eficacia con la introducción de estas variables en sus contenidos.

\section{- Conflicto de intereses.}

Los autores declaran no tener ningún conflicto de intereses.

\section{REFERENCIAS}

Álvarez-Muelas, A., Gómez-Berrocal, C., y Sierra, J. C. (2020a). Relationship of sexual double standard with sexual functioning and risk sexual behaviors: A systematic review. Revista Iberoamericana de Psicología y Salud, $17(2), \quad$ 103-116. https://dx.doi. $\mathrm{org} / 10.23923 /$ i.rips.2020.02.038

Álvarez-Muelas, A., Gómez-Berrocal, C., \& Sierra, J. C. (2020b). Typologies of sexual double standard adherence in spanish population. European Journal of Psychology Applied to Legal Context. Advance online publication.

Arce, R., Arias, E., Novo, M., y Fariña, F. (2020). Are interventions with batterers effective? A meta-analytical review. Psychosocial Intervention, 29(3), 153-164. https://doi. org/10.5093/pi2020a 11

Bandura, A. (1986). Social foundations of thought and action. En D. Marks (Ed.), The health psychology reader (pp. 23-28). Englewood Cliffs, NJ: Sage.

Baumeister, R. F., y Twenge, J. M. (2002).
Supresión cultural de la sexualidad femenina. Revisión de Psicología General, 6(2), 166 203.

Benstead, L. J. (2020). Conceptualizing and measuring patriarchy: The importance of feminist theory. Mediterranean Politics. Advance online publication. https://dx.doi.or $\mathrm{g} / 10.1080 / 13629395.2020 .1729627$

Bermúdez, M. P. , y Meléndez-Domínguez, M. (2020). Epidemiological analysis of gender violence in the European Union. Anales de psicología, 36(3), 380-385. https://doi. org/10.6018/analesps.428611

Beserra, M. A., Leitão, M. N. D. C., Fernandes, M. I. D., Scatena, L., Vidinha, T. S. D. S., Silva, L. M. P. D., y Ferriane, M. D. G. D. C. (2015). Prevalência de violência no namoro entre adolescentes de escolas públicas de Recife/Pe: Brasil [Prevalence of Dating Violence among Adolescents from Brazilian Public Schools of Recife/Pe - Brazil]. Revista de Enfermagem Referência, IV(7), 91-99. http://dx.doi.org/10.12707/RIV15006

Bringas Molleda, C., Estrada Pineda, C., Suárez Álvarez, J., Torres, A., Rodríguez Díaz, F. J., García Cueto, E., y Rodríguez Franco, L. (2017). Actitud sexista y trascendente durante el noviazgo entre universitarios latinoamericanos [Sexist and transcendent attitude during courtship between Latin American university students]. Revista Iberoamericana de Psicología y Salud, $8(1), \quad 44-55$. https://doi.org/10.23923/i. rips.2017.08.005

Borges, L. J., Heine, J. A., y Dell'Aglio, D. D. (2020). Personal and contextual predictors for adolescent dating violence perpetration. Acta Colombiana de Psicología, 23(2), 449-459. https://doi.org/10.14718/ ACP.2020.23.2.16

Cortés-Ayala, L., Flores Galaz, M., Bringas Molleda, C., Rodríguez-Franco, L., LópezCepero Borrego, J., y Rodríguez Díaz, F. J. (2015). Relación de maltrato en el noviazgo de jóvenes mexicanos: Análisis diferencial por sexo y nivel de estudios [Intimate partner violence in the relationships of Mexican youth. Differential analysis by sex and level 
of schooling]. Terapia Psicológica, 33(1), 5-12. https://dx.doi.org/10.4067/S0718$\underline{48082015000100001}$

Danube, C., Norris, J., Stappenbeck, C., Davis, K., George, W., Zawacki, T., Morrison, D., y Abdallah, D. (2016). Partner type, sexual double standard endorsement, and ambivalence predict abdication and unprotected sex intentions in a community sample of young women. Journal of Sex Research, 53(4-5), 601-613. https://dx.doi. org/10.1080/00224499.2015.1061631.

Eagly, A. H., Wood, W., \& Diekman, A. B. (2000). Social role theory of sex differences and similarities: A current appraisal. The developmental social psychology of gender, 12, 174

Eaton, A., y Rose, S. (201 1). Has dating become more egalitarian? A 35 year review using sex roles. Sex Roles, 64(1 1 - 12), 843-862. https:// dx.doi.org/10.1007/s $11199-011-9957-9$

Emmerink, P. M. (2017). Gendered sexuality. Exploring dynamics of the sexual double standard (Doctoral thesis, Utrecht University, Netherlands). Recuperado de http://dspace.library.uu.nl/bitstream/ handle/1874/355073/Emmerink. pdf? sequence $=1$ \&isAllowed $=y$

Filson, J., Ulloa, E., Runfola, C., y Hokoda, A. (2009). Does powerlessness explain the relationship between intimate partner violence and depression? Journal of Interpersonal Violence, 25(3), 400-415. hitps://dx.doi. org/10.1177/0886260509334401.

García-Cueto, E., Rodríguez-Díaz, F. J., BringasMolleda, C., López-Cepero, J., PaínoQuesada, S., y Rodríguez-Franco, L. (2015). Development of the Gender Role Attitudes Scale (GRAS) amongst young Spanish people. International Journal of Clinical and Health Psychology, 15(1), 61-68. https://doi. org/10.1016/i.ijchp.2014.10.004

García-Díaz, V., Lana-Pérez, A., FernándezFeito, A., Bringas-Molleda, C., RodríguezFranco, L., y Rodríguez-Díaz, F. J. (2018). Actitudes sexistas y reconocimiento del maltrato en parejas jóvenes [Sexist attitudes and recognition of abuse in young couples].
Atención Primaria, 50(7), 398-405. hitps:// dx.doi.org/10.1016/i.aprim.2017.04.001

Garrido-Macías, M., Valor-Segura, I., y Expósito, F. (2020). Which tactics of sexual violence predict leaving the relationship? The role of dependence towards partner. European Journal of Psychology Applied to Legal Context, 12(2), 53-60. hitps://doi. $\mathrm{org} / 10.5093 /$ eipalc2020a6

Germain, J. L. (2001). Predictors of domestic violence: Power and control versus imbalance of power and related factors. En D. S. Sandhu (Ed.), Faces of violence: Psychological correlates, concepts, and intervention strategies (pp. 337-353). Hauppauge, NY: Nova Science.

Johnson, M. P., y Ferraro, K. J. (2000). Research on domestic violence in the 1990s: Making distinctions. Journal of Marriage and Family, 62, 948-963.

Kann, L., McManus, T., Harris, W. A., Shanklin, S. L., Flint, K. H., Queen, B., ... y Lim, C. (2018). Youth risk behavior surveillanceUnited States, 2017. MMWR Surveillance Summaries, 67(8), 1-114. https://dx.doi. org/10.15585/mmwr.ss6708al

Kim, Y., Lee, E., y Lee, H. (2019). Sexual double standard, dating violence recognition, and sexual assertiveness among university students in South Korea. Asian Nursing Research, 13(1), 47-52. https://dx.doi.org/10.1016/i. anr.2019.01.003.

Martínez, J., Vargas, R., y Novoa, M. (2016). Relación entre la violencia en el noviazgo y observación de modelos parentales de maltrato [Relation between dating violence and parental maltreatment model observation]. Psychologia: Avances de la Disciplina, 10(1), 101-112. https://dx.doi. org/10.21500/19002386.2470.

Matsuda, Y., McGrath, J., y Jallo, N. (2012). Use of the sexual relationship power scale in research: An integrative review. Hispanic Health Care International, 10(4), 175-189. https://10.1891/1540-4153.10.4.175.

Moyano, N., Monge, F., y Sierra, J. C. (2017). Predictors of sexual aggression in adolescents: Gender dominance vs. rape supportive 
attitudes. European Journal of Psychology Applied to Legal Context, 9(1), 25-31. https:// dx.doi.org/10.1016/i.eipal.2016.06.001

Oliveira, Q. B. M., Assis, S. G. D., Niaine, K., y Pires, T. O. (2014). Namoro na adolescência no Brasil: circularidade da violência psicológica nos diferentes contextos relacionais [Adolescent dating in Brazil: the circularity of psychological violence in different relationship contexts]. Ciência \& Saúde Coletiva, 19, 707. 718. https://dx.doi.org/10.1590/141381232014193.19052013

Peña, F., Zamorano, B., Villarreal, K., Vargas, J., Velázquez, Y., Hernández, G., Parra, V., y Ruíz, L. (2018). Violencia en el noviazgo en jóvenes y adolescentes en la frontera norte de México [Dating violence in young people and adolescents in the northern border of Mexico]. Journal Health NPEPS, 3(2), 426-440. https:// dx.doi.org/10.30681/252610103117

Pulerwitz, J., Gortmaker, S. L., y Dejong, W. (2000). Measuring sexual relationship power in HIV/ STD research. Sex Roles, 42, 637-660. https:// dx.doi.org/10.1023/A: 1007051506972

Pulerwitz, J., Mathur, S., y Woznica, D. (2018). How empowered are girls/young women in their sexual relationships? Relationship power, HIV risk, and partner violence in Kenya. PLOS ONE, 13(7), e0199733. https://dx.doi. org/10.1371/iournal.pone.0199733

Quesada, S. G. P., Aguilera-Jiménez, N., Rodríguez-Franco, L., Rodríguez-Díaz, F. J., y Alameda-Bailén, J. R. (2020). Adolescent conflict and young adult couple relationships: Directionality of violence. International Journal of Psychological Research, 13(2), 36-48. https://doi.org/10.21500/20112084.4364

Redondo, L., Fariña, F., Seijo, D., Novo, M., \& Arce, R. (2019). A meta-analytical review of the responses in the MMPI-2/MMPI-2-RF clinical and restructured scales of parents in child custody dispute. Anales de Psicología/ Annals of Psychology, 35(1), 156-165. https:// doi.org/10.6018/analesps.35.1.338381

Reyes, H., Foshee, V., Niolon, P., Reidy, D., y Hall, J. (2016). Gender role attitudes and male adolescent dating violence perpetration:
Normative beliefs as moderators. Journal of Youth and Adolescence, 45(2), 350-360.

Rodríguez-Díaz, F. J., Herrero, J., RodríguezFranco, L., Bringas-Molleda, C., PaínoQuesada, S. G., \& Pérez, B. (2017). Validation of Dating Violence Questionnaire (DVQ-R). International Journal of Clinical and Health Psychology, 17, 77-84. https://doi. org/ 10.1016/i.ijchp.2016.09.001

Rodríguez-Franco, L., Borrego, J. L., Javier, F., Díaz, R., Molleda, C. B., Bellerín, M. A. A., \& Pineda, C. E. (2010). Validation of the Dating Violence Questionnaire, DVQ ( Cuestionario de Violencia entre Novios, CUVINO) among Spanish-speaking youth: Analysis of results in Spain, Mexico and Argentina. Anuario de Psicología Clínica y de la Salud, 6, 43-50. Sanchez, D., Fetterolf, J., y Rudman, L. (2012). Eroticizing inequality in the United States: The consequences and determinants of traditional gender role adherence in intimate relationships. Journal of Sex Research, 49/23), 168-183. https://dx.doi.org/10.1080/0 $\underline{0224499.2011 .653699}$

Sierra, J. C., \& Gutiérrez-Quintanilla, J. R. (2007). Estudio psicométrico de la versión salvadoreña de la Double Standard Scale. Cuadernos de Medicina Psicosomática y Psiquiatría de Enlace, 83, 23-30.

Sierra, J. C., Moyano, N., Vallejo-Medina, P., y Gómez-Berrocal, C. (2018). An abridged Spanish version of Sexual Double Standard Scale: Factorial structure, reliability and validity evidence. International Journal of Clinical and Health Psychology, 18(1), 69-80. $\quad$ https://dx.doi.org/10.1016/i. ijchp.2017.05.003

Ulibarri, M., Roesch, S., Rangel, M., Staines, H., Amaro, H., y Strathdee, S. (2014). "Amar te Duele" ("Love Hurts"): Sexual relationship power, intimate partner violence, depression symptoms and HIV risk among female sex workers who use drugs and their noncommercial, steady partners in Mexico. AIDS and Behavior, 19(1), 9-18. https://dx.doi. org/10.1007/s 10461-014-0772-5

Vivanco, R., Moraga, S. E., Tregear, C. R., Burgos, A. V., y Peña, A. V. (2015). 
Perpetración y victimización de la violencia en relaciones de parejas en jóvenes que cursan educación superior en la ciudad de Osorno, Chile [Perpetration and victimization of violence in young couples studying higher education in the city of Osorno, Chile]. Polis: Revista Latinoamericana, 14(40), 1-15 https://dx.doi.org/10.4000/polis. 10863

Weber, E. N., Taylor, A. R., Cantos, A. L., Amado, B. G., \& O'Leary, K. D. (2019). Exploring typology categorizations of male perpetrators: A methodology study. European Journal of Psychology Applied to Legal Context, $17(2)$, 93-97. https://doi.org/10.5093/ eipalc2019a5

Wincentak, K., Connolly, J., y Card, N. (2017). Teen dating violence: A meta-analytic review of prevalence rates. Psychology of Violence, 7(2), 224-241. https://dx.doi.org/10.1037/ a0040194

Zaikman, Y., y Marks, M. J. (2017). Promoting theory-based perspectives in sexual double standard research. Sex Roles 76, 407-420. https://dx.doi.org/10.1007/s $11199-016-$ $\underline{0677-z}$ 